EESTI NSV TEADUSTE AKADEEMIA TOIMETISED.

FOOSIKA * MATEMAATIKA

ИЗВЕСТИЯ АКАДЕМИИ НАУК ЭСТОНСКОИ ССР. ФИЗИКА МАТЕМАТИКА

PROCEEDINGS OF THE ACADEMY OF SCIENCES OF THE ESTONIAN SSR. PHYSICS * MATHEMATICS

$1985,34,1$

удк 535.5

B. ФЕДОСЕЕВ

\title{
БОКОВОЕ СМЕЩЕНИЕ ЛУЧА СВЕТА ПРИ ОТРАЖЕНИИ И ПРЕЛОМЛЕНИИ
}

V. FEDOSSEJEV. VALGUSKIIRE KULGNIHE PEEGELDUMISEL JA MURDUMISEL

V. FEDOSEYEV. LATERAL DISPLACEMENT OF A LIGHT BEAM ON REFLECTION AND REFRACTION

(Представил П. Кард)

1. В $\left[{ }^{1,2}\right]$ было предсказано явление бокового смещения луча света при полном отражении (сдвиг отраженного луча в направлении, перпендикулярном плоскости падения). Впоследствие это явление было обнаружено экспериментально $\left[{ }^{3}\right]$. Предсказание основывалось на наличии поперечной компоненты потока энергии при возникновении неоднородных волн $\left[{ }^{4}\right]$. В $\left[{ }^{5}\right]$ боковое смещение отраженного луча было вычислено с помощью метода стационарной фазы, при этом получено, что явление должно иметь место как при полном, так и при частичном отражении.

В данной работе приведены результаты расчетов боковых смещений центров тяжести отраженного и преломленного пучков света. Полученная величина бокового смещения отраженного пучка совпадает с результатами $\left[{ }^{5}\right]$.

Впервые показано наличие бокового смещения у преломленного пучка (луча) света при эллиптической поляризации.

2. Рассмотрим отражение и преломление пучка электромагнитных волн на плоской границе раздела двух изотропных сред. Геометрия задачи представлена на рисунке. Волновые векторы центральных компонент падающего $(i)$, отраженного $(\rho)$ и преломленного $(\tau)$ пучков $\vec{k}_{J}(J=i, \varrho, \tau)$ лежат в плоскости $y=0$.

Электрические векторы каждого из пучков можно представить в виде

$$
\left.\left.\vec{E}_{J}(\vec{r})=\frac{1}{2 \pi} \int_{J} \overrightarrow{d x} f(\vec{x}) \vec{\delta}_{J}(\vec{x}) e^{i\left[\omega t-\left(\vec{k}_{J}+\vec{x}_{J}\right.\right.}, \vec{r}\right)\right],-
$$

где $\vec{x}_{J}-$ двумерный вектор, $\vec{x}_{J}=x_{a} \vec{a}+v_{J} x_{b} \vec{b}_{J}, \vec{x} \equiv \vec{x}_{i}, \vec{a}-$ единичный вектор, направленный вдоль оси $0 y, \vec{b}_{J}=\left[\vec{a} \vec{k}_{J}\right] k_{J}^{-1}, v_{J}=\cos \theta_{i} \sec \theta_{J}, \theta_{i}, \theta_{\rho}$ и $\theta_{\tau}$ - углы падения, отражения и преломления, $f(\vec{x})-$ функция, нор- 
Расположение векторов, характеризующих центральные компоненты падающепо, отраженного и преломленного пучков света.

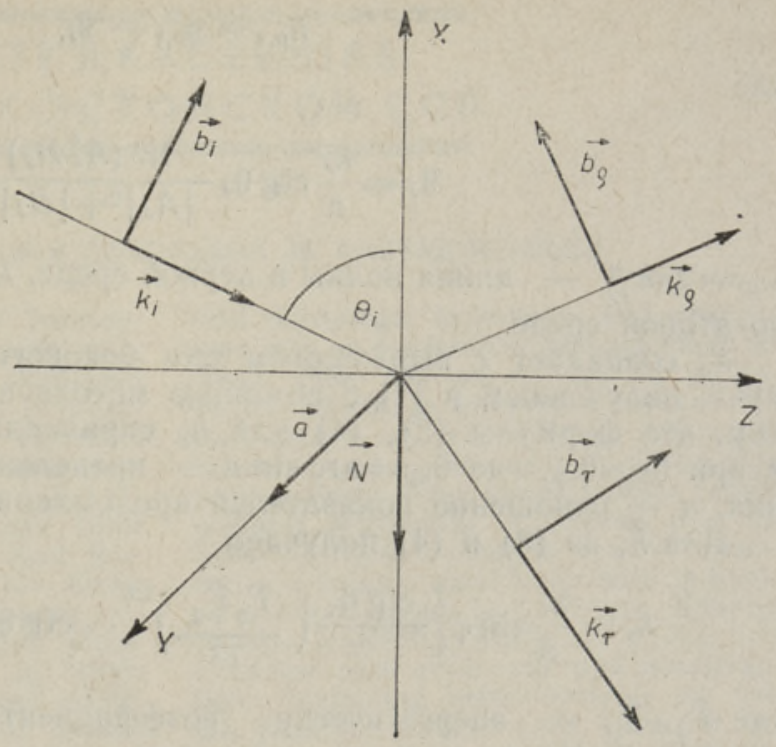

мированная на единицу, ее-полуширина $\delta$ мала по сравнению с $k_{J} . \omega-$ частота света; с точностью до членов первого порядка по $\delta k_{J}^{-1} \omega$ от $\vec{x}$ не зависит. Времена $t$ будем считать достаточно малыми для того, чтобы можно было пренебречь расплыванием пучков. $\vec{\varepsilon}_{J}(\vec{x})=A_{J}(\vec{x}) \overrightarrow{a_{J}}(\vec{x})+$ $+B_{J}(\vec{x}) \vec{b}_{J}(\vec{x}), \vec{a}_{J}(\vec{x})=\left[\vec{N},\left(\vec{k}_{J}+\vec{x}_{J}\right)\right]\left|\left[\vec{N},\left(\vec{k}_{J}+\vec{x}_{J}\right)\right]\right|^{-1}, \vec{N}-$ нормаль к плоскости раздела сред, направленная из первой (верхней) среды во вторую (нижнюю), $\vec{b}_{J}(\vec{x})=\left[\vec{a}_{J}(\vec{x}) ;\left(\vec{k}_{J}+\vec{x}_{J}\right)\right]\left|\vec{k}_{J}+\vec{x}_{J}\right|^{-1} . \quad$ Амплитуды $A_{\rho, \tau}(\vec{x})$ и $B_{\rho, \tau}(\vec{x})$ связаны с $A_{i}(\vec{x})$ и $B_{i}(\vec{x})$ формулами Френеля [ ${ }^{6}$. Без потери общности можно принять, что $A_{i}(\vec{x})-$ действительная функция,

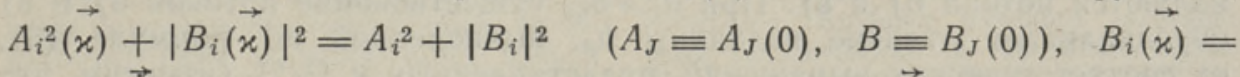
$=\left|B_{i}(\vec{x})\right| \exp (i \varphi)$. Полагаем, что $\varphi$ не зависит от $\vec{x}$, а фаза функции $f(\vec{x})$ незначительно меняется в интервале порядка $\delta$.

В каждом пучке мысленно выделим участок малой длины. $y$ координата центра тяжести участка $J$-го пучка определяется следующим образом:

$$
y_{J}^{c}=\int \overrightarrow{d r} y W_{J}(\vec{r})\left(\int \overrightarrow{d r} W_{J}(\vec{r})\right)^{-1}
$$

где $W_{J}(\vec{r})$ - плотность электромагнитной энергии $J$-го пучка $\left[{ }^{6}\right]$; интегрирование производится по объему выделенного участка.

В формуле (2) один из пределов интегрирования по переменной $\sigma=v_{J}\left(x \sin \theta_{J}+z \cos \theta_{J}\right) \quad$ зависит от положения выделенного участка. Пусть участок достаточно удален от поверхности раздела сред, тогда указанный предел может быть заменен на $\pm \infty$. В этом случае $y_{J}{ }^{c}$ от положения выделенного участка пучка не зависит, и смещение пучка при отражении и преломлении $h_{\rho, \tau}$ может быть определено как $h_{\rho, \tau}=y_{\rho, \tau}^{c}-y_{i}^{c}$.

Вычислив $y_{J}{ }^{c}$ с помощью (1) с точностью до членов первого порядка по параметру $\delta k_{J}^{-1}$, получим следующий результат: 


$$
h_{\rho, \tau}=y_{\rho, \tau}-y_{i}
$$

где

$$
y_{J}=\frac{\lambda_{J}}{\pi} \operatorname{ctg} \theta_{J} \frac{\operatorname{Im}\left(A_{J}^{*} B_{J}\right)}{\left|A_{J}\right|^{2}+\left|B_{J}\right|^{2}}
$$

$\lambda_{i, \rho}=2 \pi k_{i, \rho}^{-1}-$ длина волны в первой среде, $\lambda_{\tau}=2 \pi k_{J}{ }^{-1}-$ длина волны во второй среде.

$h_{\rho}$ совпадает с выражением для бокового смещения отраженного луча, полученным в $\left[{ }^{5}\right]$ с помощью метода стационарной фазы (заметим, что формулы (3), (4) для $h_{\rho}$ справедливы как при $\theta_{i}<\theta_{\mathrm{kp}}$, так и при $\theta_{i}>\theta_{\mathrm{kp}}$, где $\theta_{\mathrm{kp}}=\operatorname{arc} \sin n-$ предельный угол полного отражения, $n-$ отношение показателей преломления второй и первой сред). Для $h_{\tau}$ из (3) и (4) получаем

$$
h_{\tau}=\frac{\lambda_{i}}{\pi} \sin \varphi\left[\frac{\operatorname{ctg} \theta_{\tau}}{n}\left(\frac{T_{\|} T_{\perp}}{T^{2}}\right)^{1,2}-\operatorname{ctg} \theta_{i}\right] \frac{A_{i}\left|B_{i}\right|}{A_{i}^{2}+\left|B_{i}\right|^{2}},
$$

где $T_{\|}, T_{\perp}-$ энергетические коэффициенты пропускания плоских волн соответствующей поляризации $\left[{ }^{6}\right], \quad T=\left(T_{\perp} A_{i}^{2}+T_{\|}\left|B_{i}\right|^{2}\right) \times$ $\times\left(A_{i}^{2}+\left|B_{i}\right|^{2}\right)^{-1}$.-

Боковое смещение преломленного луча отсутствует при линейной поляризации. Независимо от поляризации $h_{\tau} \rightarrow 0$ при $\theta_{i} \rightarrow 0$, а при $\theta_{i} \rightarrow \theta_{\mathrm{kp}}$ величина $h_{\tau}$ составляет половину величины $h_{\rho}$.

3. Принципиальным является вопрос о физической природе боковых смещений лучей при $\theta_{i}<\theta_{\mathrm{kp}}$.

Боковые смещения лучей обусловлены потоками энергии, перпендикулярными плоскости падения (поперечными потоками). Наш анализ показал, что при отражении и преломлении волновых пакетов существуют следующие поперечные потоки (в общем сравнимые по величине): a) поток энергии неоднородных волн; б) потоки энергии однородных волн; в) интерференционный поток энергии. Причиной боковых смещений преломленного луча, а также отраженного луча при $\theta_{i}<\theta_{\text {кр }}$ являются потоки б) и в). При $\theta_{i}>\theta_{\text {kp }}$ существование потоков б) и в) обуславливает отличие величины $h_{\rho}$, вычисленной по формуле (3), от соответствующих выражений, представленных в $[2,3]$ (заметим, что последние отличаются от $h_{\rho}$ (3) на фактор $\left(1-n^{2}\right)^{-1}$ при всех значениях $\theta_{i}$ ).

Автор выражает благодарность К. К. Ребане за поддержку работы, Н. Кристофелю и П. Адамсону за обсуждения.

\section{ЛИ ТЕРА Т У Р А}

1. Федоров Ф. И. Докл. АН СССР, 105, 465-468 (1955).

2. Кристофель Н. Н. Уч. зап. Тартуск. ун-та, № 42, 94-112 (1956).

3. Imbert, C. Phys. Rev., D5, 787-796 (1972).

4. Wiegrefe, $\dot{A}$. Ann. phys., 45, 465-477 (1914).

5. Schilling, $H$. Ann. phys., 16, 122-134 (1966).

6. Борн М., Вольф Э. Основы оптики. М., «Наука», 1973.

Институт физики

Академии наук Эстонской ССР
Поступила в редакцию $11 /$ VI 1984 